\title{
Application of contrast enhanced ultrasound in gallbladder lesion: is it helpful to improve the diagnostic capabilities?
}

\author{
Wen-Tao Kong1, Hai-Yun Shen¹, Yu-Dong Qiu², Hao-Han', Bao-Jie Wen', Min Wu' \\ ${ }^{1}$ Department of Ultrasound, ${ }^{2}$ Department of Hepatobiliary Diseases, Drumtower Hospital, Medical College of Nanjing \\ University, Nanjing, China
}

\begin{abstract}
The aim of this study is to evaluate if contrast enhanced ultrasound (CEUS) can improve the differential diagnostic performance of gallbladder (GB) lesions. Materials and methods: Forty-nine patients (18 men, 31 women; mean age, $54.8 \pm 14.4$ years, range age, 22-78 years) with GB lesions (mass-forming and wall-thickened types) were enrolled in this study. All patients underwent conventional ultrasonography (US) and CEUS examination. The imaging characteristics of GB lesions were analyzed to compare the diagnostic performance of US and CEUS. The final diagnosis was obtained by histopathology. Results: There were significant differences between benign and malignant GB lesions with regards to size, shape, vascularity, the integrity and margin of GB wall and time to iso-enhancement on CEUS ( $p<0.05)$. However, no significant difference was found concerning the enhancement patterns between the two groups $(p>0.05)$. Logistic regression analysis showed that the boundary between liver and GB wall $(\mathrm{p}=0.017)$ and vascularity on color Doppler flow imaging $(\mathrm{p}=0.013)$ were two independent predictors of malignancy. The diagnostic accuracy of US could be improved in combination with CEUS (65.3\% vs $83.7 \%)$. The diagnostic accuracy of the GB wall thickening type was higher than the mass forming type. Conclusion: CEUS could improve the diagnostic performance of GB lesions, especially for wall-thickened type lesions.
\end{abstract}

Keywords: contrast enhanced ultrasound; gallbladder; carcinoma; diagnostic performance

\section{Introduction}

Gallbladder (GB) carcinoma is the most common malignant tumor of the biliary tract. The outcome of gallbladder carcinoma is poor, with an overall survival rate less than 5 years [1]. Gallbladder carcinoma in early stage confined to the mucosa is potentially curable. If there are no residual tumor cells, long-term survival in the case of complete resection is promising [2]. Unfortunately, owing to its nonspecific symptoms, the majority of patients with malignant gallbladder disease are discovered at a late stage resulting in the loss of a chance for operating. However, given the difficulty in distinguishing certain

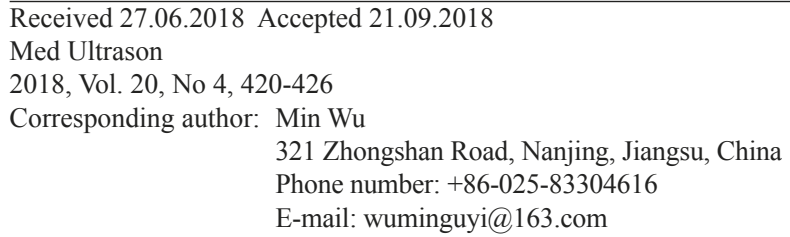

cases from gallbladder cancer before surgery, it is important to be familiar with the imaging characteristics of GB lesions. Some lesions may resemble GB carcinoma such as Xanthogranulomatous cholecystitis (XGC), adenomyomatosis (ADM), adenoma or polyp [3].

Conventional ultrasonography (US) is the first choice imaging technique used for investigating gallbladder disease with the advantages of convenience, cost effectiveness and real-time. It may provide information regarding the GB walls, lesion characteristics and adjacent liver modifications [4]. But for some GB lesions, the correct differential diagnosis may be difficult, such as motionless sludge or wall thickening of chronic cholecystitis.

Contrast enhanced ultrasound (CEUS) is frequently used for the diagnosis of focal liver lesions for its convenience, non-radiation and non-nephrotoxicity. The EFSUMB guidelines of 2011 recommended the technique for extrahepatic organs, including the biliary system [5]. It can allow assessment of GB wall perfusion, tumor microcirculation and facilitate the location of the lesion. 
The CEUS characteristics of biliary system have been reported by some other studies $[4,6,7]$.

The aim of this retrospective study was to evaluate whether CEUS in combination with conventional US can improve the diagnostic performance of GB lesions.

\section{Materials and methods}

\section{Patients}

Between February 2016 to January 2018, 49 patients with suspected gallbladder lesions were referred in our department for US examination and were retrospectively analyzed in this study. The inclusion criteria applied during the search in our database were as follows: a) patients with completed clinical, US and CEUS data; b) the presence of a parenchymatous mass inside the GB or irregular GB wall thickening. The patients unsuitable for CEUS examination such as having a recent coronary syndrome, older than 80 years or younger than 18 years and patients without histopathological results were excluded from this analysis. Twenty-four patients presented with right upper abdominal pain or discomfort and in two cases jaundice was associated. One patient complained of a progressive weight loss. The other 24 patients were asymptomatic and were detected by routine US examination. Pathologic findings were obtained from 47 cases after laparoscopic $(n=29)$ or open cholecystectomy $(\mathrm{n}=18)$. For the other 2 cases, pathological results were obtained from biopsy of liver metastasis.

Written informed consent was obtained from all patients enrolled and the study fulfilled the ethical rules of our hospital.

\section{Conventional US and CEUS examination}

Each patient was fasted for at least 8 hours before examination. US and CEUS were performed using GE LOGIQ-9 (GE Healthcare, Milwaukee, WI) and IU 22 (Philips, Bothell, WA, USA) with a $3.5-\mathrm{MHz}$ convex array abdominal probe. Conventional US was first performed to assess the size, shape and echogenicity of GB lesions. The wall of GB and adjacent liver were also evaluated. Then color Doppler flow imaging (CDFI) was used to assess the blood flow in mass, adjusted to low velocity scale of $2-10 \mathrm{~cm} / \mathrm{sec}$. CEUS was performed with a low mechanical index $(<0.10)$.A dose of $2.0 \mathrm{ml}$ (for IU 22) or $1.2 \mathrm{ml}$ (for LOGIQ E9) sulfur hexafluoride microbubbles (Sonovue, Bracco, Milan, Italy) was injected into peripheral vein in bolus followed by a flush of $5 \mathrm{ml}$ of $0.9 \%$ normal saline. There were two phases after Sonovue administration: the arterial phase, defined as 10-20 $\mathrm{s}$ after injection and the venous phase, defined as 30-45 $\mathrm{s}$ after injection [2]. Entire CEUS examination was observed for $3 \mathrm{~min}$. Another bolus was administered $20 \mathrm{~min}$ after first CEUS administration if the initial imaging was unclear. All the static or dynamic images were stored in hard disk for further analysis.

\section{Imaging analysis}

On conventional US, the lesions were divided into two types: mass-forming type and wall-thickened type. On CEUS, the enhancement pattern during arterial phase was classified into homogeneous and heterogeneous enhancement. The enhancement level was defined in comparison with the liver parenchyma at the same depth (hypoechoic, isoechoic and hyperechoic). The initial time to enhancement, time to iso-enhancement and time to hypo-enhancement were recorded. The thickness and integrity of GB wall was measured and evaluated by US and CEUS. When the continuous high echogenicity of GB wall was incomplete and enhanced inconsistently on CEUS, it was defined as wall destruction. Invasion of the adjacent liver was defined as heterogeneous enhancement (hyper- or hypo- enhancement) during the arterial phase. Wash-out was defined as hypoechogenicity during venous phase.

Consulted other references, the diagnostic criteria of a malignant tumor were as follows [6-9]: a) lesions larger than $20 \mathrm{~mm}$; b) irregular shape; c) GB wall destruction; d) liver invasion or metastasis; e) quick wash-out of contrast agent (within $60 \mathrm{~s}$ after Sonovue administration). All images were analyzed by two investigators with at least three years experience of CEUS. If there was a discrepancy between them, the question was discussed until consensus was reached.

\section{Statistical analysis}

Statistical analysis was performed using SPSS Statistics 22.0 (Chicago, IL, USA). Data were expressed as mean \pm standard deviation. Independent samples t-test was used to analyze the quantitative data. The Chisquare test and Fisher exact test were used to analyze the categorical data. The sensitivity, specificity, positive predictive value (PPV), negative predictive value (NPV), and accuracy of conventional US and combined data were analyzed. Logistic regression analysis was used to find the independent factors associated with GB lesions. Twotailed $\mathrm{p}<0.05$ was considered statistically significant.

\section{Results}

\section{Patient characteristics}

Forty-nine patients (18 men, 31 women; mean age, $54.8 \pm 14.4$ years, range age, 22-78 years) were included in this study. Final diagnosis was malignancy in 15 cases (all adenocarcinomas) and benign lesions in 34 benign cases (cholesterol polyp - 11 cases, adenoma - 7 cases, adenomyomatosis - 7 cases, Xanthogranulomatous cho- 
lecystitis in 5 cases and chronic cholecystitis in 4 cases, combined with 3 cases of motionless biliary sludge).

\section{Conventional US}

The mean diameter of malignant GB lesions was $3.08 \pm 1.15 \mathrm{~cm}$, whereas benign lesions was $1.88 \pm 0.96$ $\mathrm{cm}$, with significant difference $(\mathrm{p}=0.002)$. The number of mass-forming type cases was 37 (13 malignancies and 24 benign ones), whereas the wall-thicken type was 12 (2 malignancies and 10 benign ones). The shape of malignant lesions was more prone to be irregular than benign ones $(p=0.015)$. Also, there was a significant difference regarding to vascularity on CDFI between benign and malignant lesions $(\mathrm{p}=0.008)$. As for the $\mathrm{GB}$ wall, in $6 / 15$ malignant cases and 31/34 benign cases the wall remained continuous $(\mathrm{p}=0.001)$. The boundary between GB wall and liver was unclear in 9/15 malignant cases and $1 / 34$ benign ones $(\mathrm{p}<0.001)$.

However, there were no significant differences between benign and malignant lesions with regards to lesion type, number, echogenicity and the presence of GB stones.

\section{CEUS}

All patients received CEUS examination successfully without any adverse reaction. Forty-six GB lesions showed enhanced in arterial phase. For benign lesions, the numbers that showed hyper-enhanced, iso-enhanced and hypo-enhanced were 20,10, 1, respectively. For malignant ones, the numbers were 9,4 and 2, respectively. There was no significant difference of enhancement level between benign and malignant lesions $(\mathrm{p}=0.437)$. In terms of the enhancement pattern, there were 26 benign lesions which evidenced homogeneous enhancement and 5 appeared with heterogeneous enhancement while the numbers of malignant lesions were 8 and $7(\mathrm{p}=0.053$ ) (fig 1, fig 2). However, no enhancement was observed in 3 lesions at any time during CEUS, lesions that proved to be GB sludge after operation. Thirty-three lesions appeared hypo-enhanced during venous phase, including 23 benign and 10 malignant lesions. On CEUS, the integrity of GB wall similar to conventional US.

The median time of the lesion being iso-enhanced was $32.4 \mathrm{~s}$ for benign lesions and $45.1 \mathrm{~s}$ for malignant lesions ( $\mathrm{p}=0.013$ ). However, there was no significant difference of initial median time to enhancement and time to hypo-enhancement between these two groups.

The clinical information and US characteristics of GB lesions were summarized in Table I.
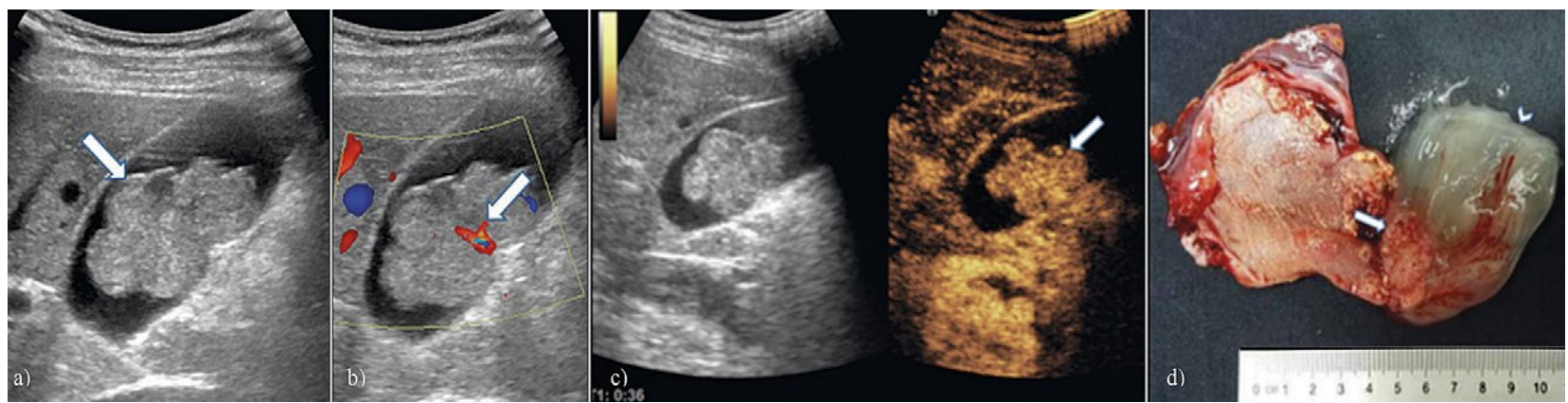

Fig 1. Images of a 46-year-old woman with gallbladder adenocarcinoma: a) B-mode US showed a homogeneously hyper-echoic lesion in the gallbladder, with irregular shape, wide stalk and low-echo (arrow); b) Blood flow signal could be detected by CDFI; c) The lesion evidenced homogeneous hyper-enhancement after contrast agent injection; d) Macropathological findings demonstrated irregular mass encrusted with mucus.

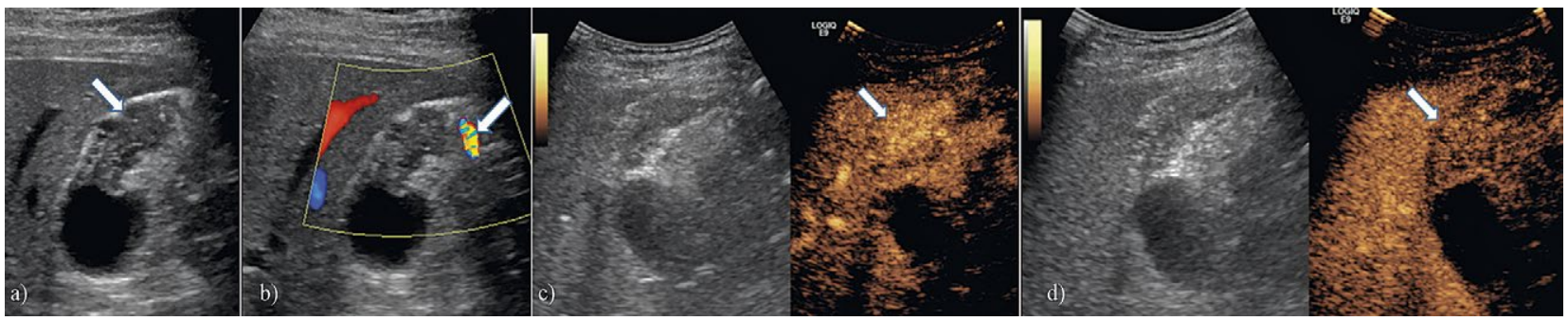

Fig 2. Gallbladder adenomyomatosis in a 48-year-old female patient. The fundus of GB wall was thickened and intramural little non-echo was detected (a). There was no blood flow on CDFI (b), only twinkling artifact (arrow). The lesion showed heterogeneous hyper-enhancement during the arterial phase (c) with hypo-enhancement during the venous phase (d). 
Table I. The clinical information and ultrasound characteristics of gall bladder lesions

\begin{tabular}{|c|c|c|c|}
\hline Characteristics & Malignant & Benign & p value \\
\hline Age (year) & 60.08 & 52.21 & 0.072 \\
\hline Gender (male/female) & $5 / 10$ & $13 / 21$ & 0.743 \\
\hline \multicolumn{4}{|l|}{ Classification } \\
\hline Mass-forming type & 13 & 24 & 0.327 \\
\hline Wall-thickened type & 2 & 10 & \\
\hline \multicolumn{4}{|l|}{ Lesion } \\
\hline Diameter $(\mathrm{cm})$ & 3.08 & 1.88 & 0.002 \\
\hline Number (single/multiple) & $9 / 4$ & $17 / 7$ & 0.919 \\
\hline Shape (regular/irregular) & $1 / 12$ & $13 / 11$ & 0.015 \\
\hline Echogenicity (hyper-/iso-hypo/mixed) & $3 / 6 / 3 / 1$ & $7 / 12 / 5 / 0$ & 0.669 \\
\hline Vascularity (yes/no) & $6 / 7$ & $1 / 23$ & 0.008 \\
\hline \multicolumn{4}{|l|}{ GB wall } \\
\hline Continuity (yes/no) & $6 / 9$ & $31 / 3$ & 0.001 \\
\hline GB wall thickening (yes/no) & $6 / 9$ & $14 / 20$ & 0.938 \\
\hline Boundary between GB and liver (clear/unclear) & $6 / 9$ & $31 / 3$ & $<0.001$ \\
\hline Gallbladder stone (yes/no) & $5 / 10$ & $7 / 27$ & 0.339 \\
\hline \multicolumn{4}{|l|}{ CEUS } \\
\hline Enhancement pattern (homogeneous/ heterogeneous/non-) & $8 / 7 / 0$ & $26 / 5 / 3$ & 0.053 \\
\hline Enhancement level (hyper-/iso-hypo/non-) & $9 / 4 / 2 / 0$ & $20 / 10 / 1 / 3$ & 0.437 \\
\hline Initial time to enhancement (s) & 16.40 & 17.77 & 0.556 \\
\hline Time to iso-enhancement (s) & 45.10 & 32.40 & 0.013 \\
\hline Time to hypo-enhancement (s) & 56.90 & 61.78 & 0.551 \\
\hline Wash-out (yes/no) & $10 / 5$ & $23 / 11$ & 0.595 \\
\hline Continuity of GB wall (yes/no) & $7 / 8$ & $33 / 1$ & $<0.001$ \\
\hline
\end{tabular}

\section{Diagnostic performance}

We correctly diagnosed 32/49 GB lesions as benign or malignant tumors on conventional US, with an accuracy of $65.3 \%$. The diagnosis was improved in combination with CEUS, with an accuracy of $83.7 \%$ (41/49). Six benign lesions were incorrectly classified as malignant, whereas 2 malignant lesions were misdiagnosed as benign (Table II).

The diagnostic performance for different types of GB lesions was as follows: for GB wall-thickened type, conventional US reached an accuracy of $91.7 \%$, with a case which could not be clearly identified. The com-

Table II. The diagnostic performance for GB lesions of conventional US and combined images

\begin{tabular}{lll}
\hline Imaging diagnosis & \multicolumn{2}{c}{ Pathologically confirmed } \\
\cline { 2 - 3 } & Malignant lesions & Benign lesions \\
\hline Conventional US & & 3 \\
malignant & 9 & 23 \\
benign & 1 & 8 \\
$\quad$ uncertain & 5 & \\
CEUS with & & \\
conventional US & & 6 \\
$\quad$ malignant & 13 & 28 \\
$\quad$ benign & 2 & \\
\hline
\end{tabular}

bined images reached an accuracy of $100 \%$. However, for mass-forming type, the accuracy was $56.8 \%$ for conventional US and $78.4 \%$ for combined images (Table III and Table IV).

The diagnostic performance for malignancy was as follows: conventional US reached sensitivity of $60.0 \%$, specificity of $67.6 \%$, positive predictive value (PPV) of $45 \%$, and negative predictive value (NPV) of $79.3 \%$. In combination with CEUS, the sensitivity, specificity, PPV and NPV were 86.7\%, 82.4\%, 68.4\% and 93.3\%, respectively. Logistic regression analysis showed that the boundary between liver and GB wall ( $p=0.017)$, and vascularity on CDFI ( $p=0.013)$ were two independent predictors of malignancy.

The misdiagnosis analysis was as follows: for conventional US, 2 cases of adenoma and 1 case of Xanthogranulomatous cholecystitis were classified incorrectly as malignant, whereas one carcinoma in situ was misdiagnosed as benign. However, the main reason for low sensitivity and specificity of conventional US was the high number of cases (13/49) with uncertain diagnosis. For the combined images, 6 benign cases were diagnosed incorrectly as GB carcinoma: 3 adenomas, 2 cholesterol polyps and one case of Xanthogranulomatous cholecystitis (fig 3). Two cases of GB carcinoma in situ 
Table III. The diagnostic performance of conventional ultrasonography for different types of GB lesions

\begin{tabular}{lll}
\hline Morphological & \multicolumn{2}{c}{ Pathologically confirmed } \\
\cline { 2 - 3 } classification & Malignant lesions & Benign lesions \\
\hline Mass-forming type & & 3 \\
$\quad$ malignant & 7 & 14 \\
benign & 1 & 7 \\
$\quad$ uncertain & 5 & \\
Wall-thickened type & & 0 \\
malignant & 2 & 9 \\
benign & 0 & 1 \\
uncertain & 0 & \\
\hline
\end{tabular}

Table IV. The diagnostic performance of CEUS in combination with conventional US for different types of GB lesions

\begin{tabular}{lll}
\hline $\begin{array}{l}\text { Morphological } \\
\text { classification }\end{array}$ & \multicolumn{2}{c}{ Pathologically confirmed } \\
\cline { 2 - 3 } & Malignant lesions & Benign lesions \\
\hline Mass-forming type & & 6 \\
malignant & 11 & 18 \\
$\quad$ benign & 2 & \\
Wall-thickened type & & 0 \\
malignant & 2 & 10 \\
benign & 0 & \\
\hline
\end{tabular}

were misdiagnosed as benign lesions. All cases diagnosed correctly by conventional US had the same results in combination with CEUS. In 9 of the 17 cases that were classified incorrectly or uncertainly by conventional US, CEUS established a correct diagnosis.

\section{Discussions}

According to the EFSUMB Guidelines of CEUS application for non-hepatic organs, CEUS was not routinely recommended for the differential diagnosis of GB lesions [5]. Recently, several papers have reported that CEUS may improve the diagnostic accuracy in combination with conventional US in GB diseases $[6,7,10]$. The 2017 version of EFSUMB Guideline updated the original point of view. In selected cases, the use of CEUS may improve the diagnostic accuracy of US [11]. Therefore, there are two purposes of this retrospective study: to establish CEUS characteristics helpful for the differential diagnosis of GB lesions and to evaluate the diagnosis performance of CEUS and related influence factors.

In our study 7 factors had significant differences between benign and malignant lesions ( 5 characteristics on conventional US and 2 on CEUS). However, logistic regression analysis showed that only 2 characteristics of conventional US were independent factors indicating a malignancy. According to these results, conventional US seems to be more useful for characterizing GB lesions. However, further analysis showed that diagnostic performance could be improved using in combination conventional US with CEUS. The main reason for low accuracy, sensitivity and specificity of conventional US in our study may be due to the high number of cases (13/49, $27 \%$ ) with uncertain diagnosis. CEUS may improve diagnostic performance by providing the information of microvasculature, especially for those lesions with indefinite diagnosis on conventional US.

We found that the morphological classification by US can affect the diagnostic performance. The diagnostic accuracy of wall-thickened type was higher. Almost all the benign cases had a distinct and intact GB wall except one lesion, which was proved to be inflammatory by pathology. Logistic regression analysis showed the indistinct boundary between the GB wall and the liver was an independent predictor of malignancy. These results were in line with other references. Xie et al found the destruction of the GB wall to be highly predictive of

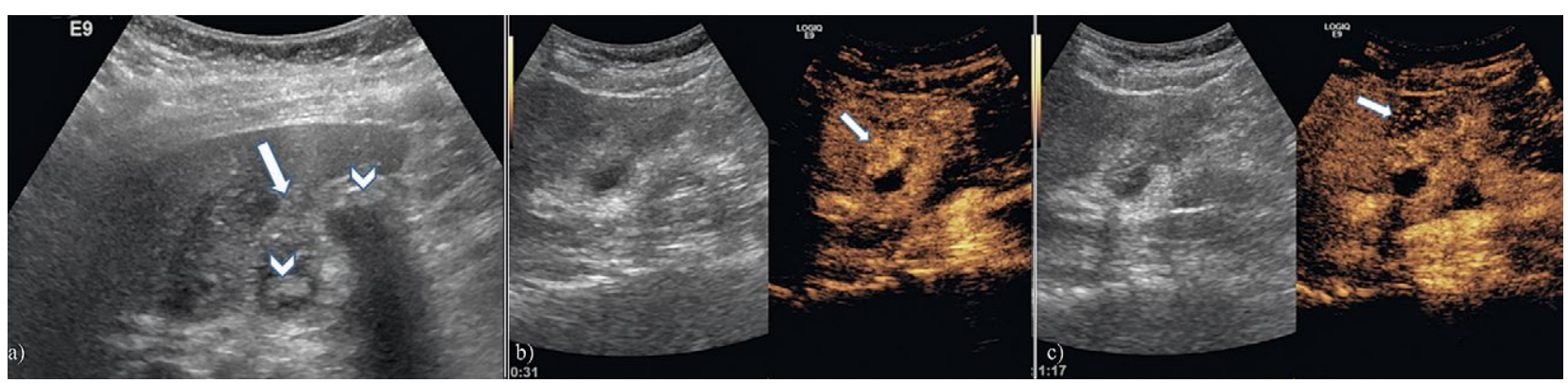

Fig 3. A 78-year-old woman with Xanthogranulomatous cholecystitis was misdiagnosed as with malignancy. On B-mode US, a markedly thickened GB wall was suspected as malignancy (a). The boundary between GB wall and liver was unclear (arrow). GB stones were also noticed (arrowheads). The wall showed heterogeneous hyper-enhancement during the arterial phase (b) and remained hypo-enhancement during the venous phase (c). However, we found destruction of the outer layer, which was proven to be an inflammatory reaction after surgery. 
malignancy with $84.8 \%$ sensitivity and $100 \%$ specificity [8]. Chen et al [12] showed that an interrupted inner layer was an independent predictor of malignancy. US can clearly depict the thickness, continuity and internal echo of GB wall. The boundary between GB wall and liver can also be evaluated. CEUS may further display the mucosal layer and serosal layer, therefore more accurately detecting the intactness of GB wall [13]. Another advantage of CEUS is the free plane scanning and real-time data acquisition [12]. All these characteristics are useful for differential diagnosis. However, both benign and malignant mass-forming lesions had a similar enhancement pattern in our study, resulting in relatively low diagnostic accuracy. The size of GB mass may be still regarded as the main diagnostic criteria for benign and malignant lesions and indication for cholecystectomy.

Some published papers demonstrated that the washout time was a useful characteristic for differential diagnosis. Malignancies were prone to have quicker washout than benign ones. Xie et al [8] proposed a cut-off for wash-out time at $35 \mathrm{~s}$, with a sensitivity of $90.9 \%$ and specificity of $87.2 \%$ in malignancy diagnosis. Serra et al [14] and Wang et al [9] set $60 \mathrm{~s}$ as an optimal cut-off for higher diagnostic accuracy in defining malignant tumors. However, our results were not in line with the above reports. We found no significant difference of wash-out time between the benign and the malignant group and the proportion of wash-out pattern was similar. This phenomenon may be related by the normal vascularization of the GB, the blood supply being provided only by the hepatic artery, which is different from that of the liver. Along with the clearance of microbubbles, almost all malignant and benign lesions will wash-out in venous phase. Also, contrast in venous phase persists for a relatively short time without the supply of the portal vein. Therefore, it may be misconceived as wash-out, especially for small lesions.

In the malignancy group of our study, two carcinomas in situ were misdiagnosed as benign on combined images. Both lesions were smaller than $2 \mathrm{~cm}$ and had US characteristics of benign lesions. The accurate diagnosis of early stage GB carcinoma is still a challenge for US. It worth noting that both US and CEUS had a low PPV for malignancy, especially for those of mass-forming type. Six benign lesions were still classified as malignant after applying contrast agent, mainly because of the quick wash-out time (shorter than $60 \mathrm{~s}$ ). It seems to indicate that using quick wash-out as a predictive predictor for malignancy may result in the false positive for those mass-forming lesions. Therefore, the role of CEUS in benign and malignant differentiation of the mass-forming GB lesions still requires evaluation.
Another advantage of CEUS is its ability to differentiate GB sludge from a tumor. GB sludge is usually detected by US and it consists of cholesterol crystals, calcium bilirubinate pigment, and other calcium salts [15]. However, the appearance of sludge may be various. Most cases are easily diagnosed but for some lesions which adhere to GB wall tightly, the differential diagnosis from a tumor could be difficult. Almost all papers pointed out CEUS had a high sensitivity and specificity for this differentiation $[4,14,16]$. Our research had the same result. Due to a lack of blood supply, three cases confirmed to be GB sludge pathologically appeared non-enhanced during arterial and venous phase.

Our study has some limitations that must be underlined. First, the number of cases was small and only a small number of cases with malignancy was included in this study. Therefore, we did not discuss further with regard to lesions' size and type. Second, it was a retrospective study. Although two investigators with three years experience of CEUS analyzed the images, CEUS features were not analyzed using quantitative analysis software so the interpretation may be subjective. Third, the morphological classification of lesions was not very accurate. Some cases classified as mass-forming type may be combined with thickened GB wall. This can lead to statistical bias.

In conclusion, CEUS could improve the diagnostic sensitivity of GB lesions by displaying microvasculature, thus reducing the cases with uncertain diagnosis by conventional US. GB lesions classified as wall-thickened type were relatively easy to diagnose because of the clear appearance of wall on conventional US and CEUS. Considering there were overlaps of enhancement pattern, those lesions of mass-forming type may not be benefit from CEUS as a routine imaging examination. CEUS was particularly useful for the differentiation between GB tumors and sludge.

Acknowledgement: This study was funded by the National Natural Science Foundation of China (81671701) and the Nanjing Medical Science and technique Development Foundation (QXR17011)

\section{Conflict of interest: none}

\section{Reference}

1. Shukla SK, Singh G, Shahi KS, Bhuvan, Pant P. Staging, Treatment, and Future Approaches of Gallbladder Carcinoma. J Gastrointest Cancer 2018;49:9-15.

2. Park TJ, Ahn KS, Kim YH, Kim TS, Hong JH, Kang KJ. The optimal surgical resection approach for T2 gallbladder 
carcinoma: evaluating the role of surgical extent according to the tumor location. Ann Surg Treat Res 2018;94:135-141.

3. Golse N, Lewin M, Rode A, Sebagh M, Mabrut JY. Gallbladder adenomyomatosis: Diagnosis and management. J Visc Surg 2017;154:345-353.

4. Gerstenmaier JF, Hoang KN, Gibson RN. Contrast-enhanced ultrasound in gallbladder disease: a pictorial review. Abdom Radiol (NY) 2016;41:1640-1652.

5. Piscaglia F, Nolsøe C, Dietrich CF, et al. The EFSUMB Guidelines and Recommendations on the Clinical Practice of Contrast Enhanced Ultrasound (CEUS): update 2011 on non-hepatic applications. Ultraschall Med 2012;33:33-59.

6. Zhang HP, Bai M, Gu JY, He YQ, Qiao XH, Du LF. Value of contrast-enhanced ultrasound in the differential diagnosis ofgallbladderlesion. WorldJGastroenterol2018;24:744-751.

7. Yuan HX, Cao JY, Kong WT, Xia HS, Wang X, Wang WP. Contrast-enhanced ultrasound in diagnosis of gallbladder adenoma. Hepatobiliary Pancreat Dis Int 2015;14:201-207.

8. Xie XH, Xu HX, Xie XY, et al. Differential diagnosis between benign and malignant gallbladder diseases with real-time contrast-enhanced ultrasound. Eur Radiol 2010;20:239-248.

9. Wang W, Fei Y, Wang F. Meta-analysis of contrast-enhanced ultrasonography for the detection of gallbladder carcinoma. Med Ultrason 2016;18:281-228.
10. Zhuang B, Li W, Wang W, et al. Contrast-enhanced ultrasonography improves the diagnostic specificity for gallbladder-confined focal tumors. Abdom Radiol (NY) 2018;43:1134-1142.

11. Sidhu PS, Cantisani V, Dietrich CF, et al. The EFSUMB Guidelines and Recommendations for the Clinical Practice of Contrast-Enhanced Ultrasound (CEUS) in Non-Hepatic Applications: Update 2017 (Short Version). Ultraschall Med 2018;39:154-180.

12. Chen LD, Huang Y, Xie XH, et al. Diagnostic nomogram for gallbladder wall thickening mimicking malignancy: using contrast-enhanced ultrasonography or multi-detector computed tomography? Abdom Radiol (NY) 2017;42:24362446.

13. Tang S, Wang Y, Wang Y. Contrast-enhanced ultrasonography to diagnose gallbladder perforation. Am J Emerg Med 2013;31:1240-1243.

14. Serra C, Felicani C, Mazzotta E, et al. CEUS in the differential diagnosis between biliary sludge, benign lesions and malignant lesions. J Ultrasound 2018;21:119126.

15. Shaffer EA. Gallbladder sludge: what is its clinical significance? Curr Gastroenterol Rep 2001;3:166-173.

16. Sparchez Z, Radu P. Role of CEUS in the diagnosis of gallbladder disease. Med Ultrason 2012;14:326-330. 